\title{
Mallarmé et les paradoxes de la vanité
}

Dans sa vie d'artiste Mallarmé a constamment balancé entre les extrêmes : ambition et impuissance créatrice, idéal et réalité, hauteur d'un aristocrate d'esprit et modestie d'un poète incompris. D'ailleurs, le recours au paradoxe semble nécesaire à qui veut saisir cette " obscurité lumineuse » mallarméenne, un message poétique dissimulé derrière des inventions syntaxiques où la référentialité du langage s'efface en vue d'une «notion pure » qui illustre le mieux le caractère autoréflexif du texte. En raison du "double état de la parole ", serait-il donc possible de considérer le double voire triple état de la vanité, ce concept qui se révèle particulièrement fécond pour la création mallarménne, tout en caractérisant une attitude qui (in)définit le poète face à son public et à lui-même ? La notion recèle en effet une contradiction de plus, car Mallarmé semblait prendre ses distances envers le paraître, sans pour autant cesser d'aspirer à une excellence littéraire. Creuser les divers aspects de la vanité mallarméenne, c'est, paraît-il, approcher le destin du poète et les principes mêmes de sa création.

\section{Le poète entre vanité et découragement}

Tout d'abord, il est nécessaire d'analyser le personnage de Mallarmé à travers la notion de vanité qui renvoie à " un sentiment d'orgueil fondé sur la satisfaction de la possession » (Verlet, 2001, p. 8) : celle des biens matériels ou du plaisir des sens, mais aussi de la gloire et du beau que l'on croit atteindre. Dans l'introduction à l'enquête Sur l'évolution littéraire (1891) Jules Huret décrit Mallarmé comme un homme

Anna Opiela-Mrozik - maître de conférences à l'Institut d'Études romanes de l'Université de Varsovie. Adresse pour correspondance : Institut d'Études romanes de l'Université de Varsovie, ul. Dobra 55, 00-312 Varsovie, Pologne ; e-mail : am.opiela@uw.edu.pl 
charmant « en qui l'on devine un immarcescible orgueil, planant au-dessus de tout, un orgueil de dieu ou d'illuminé » (Mallarmé, 2003, p. 697). Voici le maître des célèbres mardis de la rue de Rome qui donnait à ses visiteurs une sorte de spectacle en les exorcisant avec sa voix conjuguée à une attitude bien étudiée et une intelligence supérieure. Dans sa capacité de faire perdre à l’objet la référentialité au profit de l'essence, il apparaissait aux jeunes enthousiastes de la poésie comme un "prestidigitateur de la parole » (Anderson, 2002, p. 30). Si, à cette époque-là, Mallarmé jouissait d'une renommée établie dans le monde littéraire ce qui, d'autant plus, contribuait à créer son mythe toujours vivant, ce n'est que dans les années de jeunesse, au seuil de sa carrière, qu'il exprimait son vif désir d'atteindre la gloire poétique. En se mettant au travail sur Hérodiade, un sujet longtemps médité, il n’admettait qu'une optique binaire, d'où cet aveu fait à Henri Cazalis : « je veux - pour la première fois de ma vie - réussir. Je ne toucherai plus jamais à ma plume, si j’étais terrassé " (Mallarmé, 1998, p. 663). D’un côté, la nouveauté que recherchait Mallarmé à travers ses vers faisait naître chez lui une sorte d'effroi, de l'autre, la certitude de l'originalité faisait valoir son œuvre à venir. Le poète débutant, Mallarmé aurait-il donc envisagé de tirer vanité de ses textes?

Il n'est pas à nier que pour ces critiques de l'époque qui ne le jugeaient pas fou, il apparaissait pourtant comme un poète mystificateur dont l' " extravagance voulue " s'exprimait par des vers incompréhensibles. Un surréaliste avant la lettre, Mallarmé aurait donc visé à se moquer de ses lecteurs. Sinon, ce « rimeur biscornu » (Marchal, 1998, p. 63) restait au moins une énigme avec laquelle il était impossible de discuter, à moins qu'on puisse citer, sans aucun commentaire, ses vers musicaux (Marchal, 1998, p. 51). Tous soulignaient la nouveauté qui, comme a noté Verlaine dans Les Poètes maudits, "fit scandale dans les journaux " (Marchal, 1998, p. 65). Ce n'étaient que lui et quelques autres qui ont saisi l'objectif artistique du « style volontairement excessif et maniéré, parfois obscur, de Stéphane Mallarmé » (Marchal, 1998, p. 31), le poète accusé, par la plupart des commentateurs, de la mauvaise foi nuancée de prétention. Il serait pourtant intéressant de relever, dans cette multitude des opinions, celle de Gustave Lanson, ce maître de la critique universitaire qui a rédigé un long article sur Mallarmé en 1893, quand la notoriété du poète était déjà bien reconnue. Or, que Lanson indique l'inintelligible comme ce qui est le plus précieux chez Mallarmé, tout en étant le premier défaut de sa création, il le fait en refutant, en même temps, l'argument de la vanité du poète. Malgré toute ironie que contient son discours, Lanson semble avoir saisi l'essentiel de la poétique mallarméenne :

Car M. Mallarmé n'est pas inintelligible, comme nous le sommes tous parfois, par impuissance, ou par ignorance, faute de maittriser ou notre pensée ou la langue. Il n’est pas inintelligible non plus par vanité, par affectation de profondeur ou d'esprit, pour étonner les sots et les badauds : il n'entortille pas sa pensée pour en hausser le prix. Son cas est moins vulgaire. Il est inintelligible par une sublime folie idéaliste, qui fait penser à certains grands mystiques. Il y a de la sainteté dans cette folie. (Marchal, 1998, p. 273) 
C’est la folie, par laquelle Mallarmé ne cesse d'entrevoir l'idéal, qui se trouve à l'opposé de l'autosatisfaction impossible à atteindre. Aussi, peut-être, ne faudrait-il pas sétonner en lisant le dernier souhait du poète formulé dans sa lettre à la femme et à la fille, à la veille de sa mort. Dans cette espèce de testament noté au sortir d'" une vie d'artiste sincère ", il demandait de brûler tous ses papiers inédits, car « il n'y a pas là d'héritage littéraire " (Mallarmé, 1998, p. 821). Voici l'aveu d'un simple et fidèle serviteur de l'Art qui, à la manière d'un saint, ne tiendrait guère à sa gloire posthume. Parmi les textes qu'il voulait conserver, se trouve quand même Hérodiade, la pièce finalement inachevée et dont l'histoire témoigne d'une hésitation qui accompagnait Mallarmé en permanence. Au cours des années pendant lesquelles il arrêtait et reprenait le travail sur ce texte, le poète a formulé bien des idées contradictoires qui révèlent sa lutte intérieure entre le désir de splendeur littéraire et le découragement face à l'échec. Plein d'enthousiasme et d'ambition au moment de se mettre à l'œuvre, il s'est ensuite heurté à une inspiration défaillante ainsi qu'aux soucis de la vie quotidienne qui le détournaient de son travail. Après l'abandon du projet, ce qui a entraîné « une grande douleur et une humiliation » ("À Henri Cazalis », 1998, p. 676), le poète a repris son texte avec un désir exagéré de réussite et une valeur démesurée attachée à sa création : "Ah ! ce poème, je veux qu'il sorte, joyau magnifique, du sanctuaire de ma pensée ; ou je mourrai sur ses débris !" ("À Henri Cazalis », 1998, p. 684). La correspondance de l'année 1866, l'époque de travail sur Hérodiade, montre bien l'ampleur du Rêve de perfection qui, même si tout l'indique, semble pourtant être loin de la vanité du poète :

J'ai écrit l'ouverture musicale, [...] mais je puis dire sans présomption qu'elle sera d'un effet inouï [...]. Il me faudra trois ou quatre hivers encore, pour achever cette ouvre, mais j'aurai enfin fait ce que je rêve être un Poëme - digne de Poë et que les siens ne surpasseront pas. (« À Henri Cazalis », 1998, p. 696)

Si Mallarmé projette avec fermeté l'accomplissement de ses aspirations artistiques, il ne cesse quand même d'exprimer son incertitude quant à la valeur de ses textes, en cherchant en même temps à vaincre ses doutes. L'itinéraire poétique de Mallarmé s'identifie donc à un permanent va-et-vient entre le haut et le bas, l'assurance et la crainte, l'élan immodéré vers la gloire et l'état d'un artiste en train de s'autodévaloriser ${ }^{1}$. Il suffit de mentionner que Mallarmé n'est pas arrivé à mener à bien le rêve d'Hérodiade: ce n'est que dans les dernières années de sa vie qu'il s'est remis à « ces vieux vers » («À Henri Cazalis », 1998, p. 754), en envisageant leur publication

1. Il parait intéressant, dans ce contexte, de reprendre les réflexions de Paul Valéry sur les différences entre la vanité et l'orgueil. Ce dernier serait fondé sur " un mécontentement permanent de soi » (1974, p. 1413) et se réduirait à " un reproche et un blâme perpétuels » (1974, p. 1403) à l'égard de soimême. Sans être vaniteux, Mallarmé pourrait donc être qualifié d'un orgueilleux qui, en dépit des difficultés, ne cesse de s'autodiscipliner au nom d'un rêve idéaliste. 
prochaine, qui cependant n'a jamais abouti. L'ampleur idéaliste de cette œuvre majeure aurait-elle donc dépassé les moyens du poète aux prises avec un découragement constant ? Ou, au contraire, dans les retours succesifs au texte faut-il voir du désir narcissiste exprimé à travers le personnage d'Hérodiade dont le nom même indique son caractère précieux?

\section{La Poésie éprise d'elle-même}

C'est dans des réponses, pleines d'ironie ou réduites au silence, données par Mallarmé aux questions de différentes enquêtes, qu'on voit bien « ce mélange curieux de modestie et d'orgueil qui le caractérisait " (Millan, 2014, p. 371). Ce que d'ailleurs lui reprochaient plusieurs critiques pour qui les titres mêmes de ses textes, comme Divagations, relevaient d'une " orgueilleuse modestie " (Marchal, 1998, p. 410). Plus encore, dans l'un de ses premiers textes, "Hérésies artistiques. L'art pour tous " (1862), Mallarmé a fait à ses confrères un appel révoltant : « Ô poëtes, vous avez toujours été orgueilleux ; soyez plus, devenez dédaigneux!» (2003, p. 364). Selon la suggestion du titre, la démocratisation de l'art relève de l'impossible, surtout s'il s'agit de la poésie, « un seul art, [le] plus grand » dont le créateur « doit rester aristocrate». Par ailleurs, Mallarmé évoque l'idée de "vanité moderne " qui se trouve à l'origine d'un mythe égalitaire de son époque et qui se ramène au "titre vide de citoyen » (2003, p. 361).

Que cet élitisme catégorique de Mallarmé soit nuancé par le fait que son article de jeunesse a été préparé pour la revue L'Artiste, dont les gôuts parnassiens n’étaient pas à nier, il n'en reste pas moins vrai que le texte marque un point important dans l'esthétique mallarméenne qui visait à la sacralisation de l'art. Si celui-ci se situe à un pôle opposé à la foule qui reste "à la vaine couche suffisante d'intelligibilité » (Divagations, Mallarmé, 2003, p. 230), c'est qu'il voit s'attribuer, par un geste de poète, le concept de vanité. L'idéal de la poésie mallarméenne se trouve illustrée par l'attitude d'Hérodiade qui réfute ainsi tous les arguments de sa Nourrice :

Oui, c'est pour moi, pour moi, que je fleuris, déserte!

[...]

Je me crois seule en ma monotone patrie,

Et tout, autour de moi, vit dans l'idolâtrie

D'un miroir qui reflète en son calme dormant

Hérodiade au clair regard de diamant. (1998, p. 21)

Voici donc un art qui se donne à lui-même, considéré à travers « une esthétique de miroitement » (Bayle, 2014, p. 139) héritée de Baudelaire. L'art véritable n'existe en effet que grâce à la vanité, si bien que son autosatisfaction affecte également le poète qui avoue à Cazalis : " Pour moi la Poësie me tient lieu de l'amour, parce qu'elle est 
éprise d'elle-même et que sa volupté d'elle retombe délicieusemen[t en] mon âme " (1998, p. 715). Dans son attitude peu vaniteuse, Mallarmé a donc choisi de subir et de servir la vanité de l'Art, ce qui a, en effet, contribué à réduire ou bien presque à abolir une envie de gloire personnelle. Dans ses « Notes" sur Mallarmé publiées dans La Vogue en 1886, Teodor de Wyzewa a bien caractérisé la nature de la prétendue «folie " mallarménne : il s'agirait de celle d'un Artiste véritable qui a renoncé même à la Gloire. Ayant placé Mallarmé dans la lignée de Beethoven, Wyzewa a vu en lui un créateur joyeux, tel un fou de dieu de l'Art, qui « a seul préféré toujours, à tous les argents, à toutes les gloires, la création libre et consciente d'une vie artistique» (Marchal, 1998, p. 114-115).

La question de création revêt pour Mallarmé une dimension eschatologique car " tout est vain en dehors de ce rachat par l'Art » ("À Maurice Barrès », Mallarmé, 1998, p. 786). Dans son éloge du maître des Parnassiens, Théodore de Banville, nommé « musicien des mots ", Mallarmé parle de la Poésie en tant que d'un édifice grandiose qu'il est important de défendre car sans lui «tout serait vain" (Divagations, 2003, p. 143). Il semble que c'est pour cette raison, à plusieurs reprises, le poète avouait sa haine d'écrire et de répondre aux lettres, cette tâche détestée qui le fatiguait et détournait de l'Art. En accordant « cette concession à la réalité » ("À Villiers de l'Isle-Adam », 1998, p. 687), Mallarmé croyait retomber de son asile artistique dans l'insupportable banalité de la vie. Plus encore : l'Art lui assurait l'unique contrepoint aux problèmes politiques de l'époque. C'est lors de son séjour à Londres qu'il a exprimé ses désillusions sur ce point-là, en affirmant en même temps la conviction qu' « il n'y a de vrai, d'immuable, de grand, et de sacré que l'Art. Toutes les vaines disputes politiques passent, n'ayant rien d'absolu en elles " ("À Henri Cazalis », 1998, p. 650). S'il prenait ses distances envers ces dernières, c'est aussi en raison de la vanité, ce trait de caractère qu'il dénonçait chez les ouvriers participant au meeting en faveur de la Pologne.

Voici donc un paradoxe crucial de l'œuvre malarméenne qui s'appuie sur l'originalité du concept de vanité : d'un côté, la poésie repliée sur elle-même se complaît dans une autoréflexion à outrance, mais de l'autre, c'est de cette façon qu'elle protège le créateur, dans une certaine mesure, contre un sentiment de vanité, c'est-à-dire de la futilité des choses du monde. Par ailleurs, afin de donner un sens à sa vie, le poète en quête du "Grand CEuvre " déclarait dans une lettre à Verlaine sa volonté de "sacrifier toute vanité et toute satisfaction " (1998, p. 788), renonçant ainsi à lui-même au nom du poème, "rendu à lui-même et privé de tout autre sujet ", qui " expose le mystère de sa nécessité et en chante l'émerveillement " (Delègue, 2001, p. 1429). Si le travail poétique de Mallarmé s'accompagne d'un plaisir de satisfaction, c'est au nom de la perfection visée, comme il avoue, toujours à Verlaine, après avoir reçu ses Poèmes saturniens : « Mais votre livre est dans toute sa beauté et l'acception romantique, un premier volume, et qui m'a fait, bien des soirées, regretter ma vanité de ne livrer mon œuvre qu'à la fois, parfait, et quand je ne pourrai plus que décroître" (1998, p. 710-711). Le rêve, finalement échoué, du poète 
idéaliste qui désirait publier son Hérodiade dans l'intégralité du texte, «tout d'un bloc » et non en fragments, constitue donc la seule impulsion à ce que Mallarmé appelle la vanité, même s'il s'agirait plutôt d'un orgueil purement artistique aspirant à la beauté et à la vérité.

\section{De la vanité du langage au vide créateur de la poésie}

Néanmoins, plusieurs poèmes mallarméens sont marqués par une fausseté délibérée : que ce soit " un subtil mensonge " dans "Autre éventail de Mademoiselle Mallarmé » (1998, p. 31), un " air de mensonge " qui résonne dans la voix annonçant la mort de la Pénultième ("Le Démon de l'analogie », 2003, p. 87) ou le dernier aveu d'Hérodiade qui change le sens de tout son discours : "Vous mentez, ô fleur nue de mes lèvres !» $(1998$, p. 22). Comme remarque à juste titre Annick Ettlin, le dévoilement des mensonges qui met en question l'authenticité de la poésie, tout en légitimant son union avec le faux, renvoie à une « mémorable crise " mentionnée vers la fin du Coup de dés (voir Ettlin, 2017, p. 184-185). Il s'agit évidemment de la célèbre crise métaphysique des années 1860, qui a fait découvrir à Mallarmé l'existence du Néant. Celui-ci a pris la place de Dieu et a rendu au poète la conscience de la fragilité ou de la vanité de l'homme, selon une acception proche de celle de Pascal : " nous ne sommes que de vaines formes de la matière ", constate-t-il dans une lettre à Cazalis datée de 1866 (1998, p. 696). Cependant, la profession de la foi matérialiste n'est qu'une invitation à " dématérialiser la littérature " (Gallardo, 1998, p. 11), ce qu'il a fait dans Hérodiade. En effet, c'est lors du travail sur un poème de l'idéalité et de la désincarnation (la Nourrice évoque le "mystère vain » de l'être d'Hérodiade, 1998, p. 20), à force d'entrer plus profondément dans le vers, que Mallarmé « est allé jusqu'aux fondements du langage et de la signification » (Benoit, 2001, p. 12).

Ayant rejeté Dieu et le Verbe créateur, le poète a dû se rendre compte de la vanité du langage qui restait toujours son unique référent. Dans sa volonté de se donner le spectacle de la matière en se projetant dans le Rêve et en prononçant des « glorieux mensonges » face au Néant («À Henri Cazalis », 1998, p. 696), Mallarmé a été obligé de trouver une solution : concilier le Rêve et le Vide (1998, p. 732)2. Et c'est là qu'il faudrait chercher la source essentielle de l'obscurité mallarméenne : dans le monde privé d'une instance transcendentale et menacé par le non-sens, le langage devrait jouer sur le vide même qui déboucherait sur un sens atteint grâce au "mirage interne des mots mêmes" (1998, p. 731). Se formule ainsi un nouveau paradoxe : tout en évitant l'attitude d'un vaniteux, Mallarmé a délibérement choisi de révéler et d'ex-

2. Plus encore : le vide, telle une table rase, s'est avéré nécessaire pour relever une idée pure de l'expérience du Néant. Mallarmé précise ainsi à Villiers les détails de sa découverte idéaliste : "pour garder une notion ineffaçable du Néant pur, j'ai dû imposer à mon cerveau la sensation du vide absolu » (1998, p. 724). 
ploiter l'illusion du langage en voilant son sens. À en croire Paul Bénichou, malgré l'ambiguité inscrite dans cette décision, Mallarmé considérait que "la parole claire serait vantardise, parce qu'elle se targuerait de vertus qu'elle n'a pas, communicabilité et connaissance ; et elle serait courtisanerie, en mendiant à l'homme un simulacre d'écoute et au monde une lumière qu'il lui refuse » (1998, p. 59).

Selon la logique mallarméenne, la vanité de la parole, dont la conséquence inévitable était la libération de la poésie de tous ses sujets, a dû entraîner l'effacement du moi créateur, la première instance du texte. Le poète lui-même, sorti de la crise de Tournon, a donc découvert sa propre vanité conçue dans un sens proche de celui des Vanités picturales. Ayant ainsi délivré le poème de toute attache extérieure, Mallarmé n’a pas hésité à constater dans "Crise de vers » :

L'œuvre pure implique la disparition élocutoire du poëte qui cède l'initiative aux mots, par le heurt de leur inégalité mobilisés ; ils s'allument de reflets réciproques comme une virtuelle traînée de feux sur des pierreries, remplaçant la respiration perceptible en l'ancien souffle lyrique ou la direction personnelle enthousiaste de la phrase. (2003, p. 211)

Bien que les mots restent toujours un matériau de la littérature et, par la nature même du signe linguistique, continuent à désigner une réalité extra-poétique, Mallarmé se situe dans le sillage de Poe et propose dans la formule bien connue de «peindre, non la chose, mais l'effet qu'elle produit " (« À Henri Cazalis », 1998, p. 663), en insistant sur l'inanité de la parole, ce terme qui, au sens figuré, signifie le vide et la vanité (l'adjectif « vain ", venant du latin " vanus » qui désigne ce qui est vide, sans substance ; voir Lanini, 2006, p. 12). Ainsi, arrive-t-on à ce qui constitue pour Mallarmé une vérité esthétique : c'est la sincérité de l'idée qui prévaut sur l'authenticité des faits. Dans la création fondée sur le mensonge, c'est le vide même, qu'illustre bien « le vide de signification » de la Pénultième ("Démon de l'analogie ", 2003, p. 87), qui peut se trouver à l'origine de la fascination qu'engendre un mystère poétique (voir Ettlin, 2017, p. 183).

Selon la définition mallarméenne, le vide est donc mis en œuvre en vue d'atteindre à l’objectivité de la poésie. Les mots, dont la référentialité a été réfusée, se reflètent comme des bijoux ornant Hérodiade, en faisant preuve de la vanité du poème qui, " fermé sur lui-même, autosatisfait, [...] jouit de son évidence accessible à nul autre regard que le sien »(Delègue, 2001, p. 1425). Si le sens des mots est condamné à son évacuation, il n'en reste que la vacuité sonore du langage qui, paradoxalement, devrait créer une nouvelle signification. Voici donc l'avènement d'une esthétique de l'absence ou de la vanité, ce synonyme du vide, qui réunit un moyen et un objectif dans l'opération de " creuser le vers ", conduisant à la brisure de celui-ci. 


\section{4. "Vains coquillages, sonores et vides "}

C'est par cette notion péjorative que Proust, alors âgé de 25 ans, dans l'essai « Contre l'obscurité » (1971, p. 390), dénonçait les clichés rhétoriques des jeunes poètes. Cependant, dans le cas de Mallarmé, il serait difficile de trouver un terme plus approprié pour saisir la nature et la valeur de son écriture qui a rendu la vanité sensu largo son principe créateur et organisateur. Voici une caractéristique du "ptyx » qui, environné de mots vains, illusoires et condamnés à la disparition, contribue à créer une sorte de symphonie miroitant dans l'exaltation du néant :

Sur les crédences, au salon vide : nul ptyx,

Aboli bibelot d'inanité sonore,

(Car le Maître est allé puiser des pleurs au Styx

Avec ce seul objet dont le Néant s’honore). («Ses purs ongles... », 1998, p. 37)

On connait le goût de Mallarmé pour les beaux objets et la sensibilité aux questions de décoration d'intérieur. Dans le "Sonnet en -yx », le salon n'est meublé que de crédences, probablement anciennes, mais ce qui devait l'orner et combler son espace, notamment des bibelots, se ramène au souvenir d'un objet inexistant dont il n'est resté qu'un nom vide de sens. Néanmoins, c'est sa sonorité qui est mise en valeur dans une sorte d'incantation du Néant. Une musicalité, brusquement surgie du vide et convoquée par la rime, constitue donc un facteur générateur de sens même si, en réalité, des sons répétés en écho, comme sortis d'une caisse creuse, sont voués à revenir au vide, en se muant instantanément en silence. C’est ainsi que le ptyx devient un nouveau " 'objet musical' rêvé, idéal, suggestif et sonore comme la musique, verbal et rimique comme un mot poétique, soit un 'objet musical' inouï dans tous les sens du terme » (Albrecht, 2016, p. 93).

La réalité du ptyx ne change pourtant pas. Il reste vide à la manière de l'ancien instrument du sonnet «Une dentelle s'abolit... », où se produit le même phénomène du jeu sonore qui invente une signification :

Mais chez qui du rêve se dore

Tristement dort une mandore

Au creux néant musicien. (1998, p. 42)

Tout se passe, en effet, au niveau du vide dont découle un sens saisi dans « l'instant poétique ", selon le terme proposé par Gaston Bachelard. L'instrument incapable de rendre un son acoustique se trouve valorisé par la vacuité de la sonorité poétique : la rime qui est renforcée par une rime intérieure, cette représentation sonore de l'instrument même ( «-ment dore») qui sonne le creux, s'efface finalement dans l'absence.

Le vide acoustique se dégage également du jeu du faune qui, dans sa plainte amoureuse et la vaine tentative de faire revenir les nymphes, se sert de deux « creux roseaux 
domptés par le talent » (Mallarmé, 1998, p. 23). Grâce à son génie créateur, le faunepoète compose sur sa flûte une rêverie qui est celle du vide instauré après la fuite des femmes. Au moyen d'un motif musical fondé sur un son unique qui, par son bourdonnement permanent, engendre une illusion de la musique (" Une sonore, vaine et monotone ligne », p. 24), Mallarmé suggère l'épiphanie du néant, car « ce n'est pas l'objet du désir en tant que corps qui retient le faune mais au contraire sa disparition et le creux que cette disparition laisse dans l'opacité du monde » (Bougault, 1998, p. 69) ${ }^{3}$. La réalité matérielle étant abolie, c'est donc la projection de l'idée que lance le faune en vue d'une reconstruction idéale mais qui, relayée aussi par son aspect parodique, ne cesse de rester vaine :
Ainsi, quand des raisins j'ai sucé la clarté,
Pour bannir un regret par ma feinte écarté,
Rieur, jélève au ciel d'été la grappe vide
Et, soufflant dans ses peaux lumineuses, avide
D’ivresse, jusqu'au soir je regarde de travers

Ô nymphes, regonflons des SOUVENIRS divers. (1998, p. 24)

Le geste de souffler dans les peaux vidées de la grappe, de même que le désir de " regonfler » des souvenirs, ont la même fonction : jouir de ce qui relève de l'absence. Mais force est de remarquer que, dans le soliloque du faune, la vanité, dans le sens d' " outrecuidance" (Davies, 1988, p. 64), possède la faculté de bouleverser la réalité : « Ô bords siciliens d'un calme marécage / Qu'à l'envie des soleils ma vanité saccage " (1998, p. 23). C'est afin de relever l'autosatisfaction du poète que la nature est convoquée à se conformer à son état par l'intermédiaire de l'instrument, ce produit de la nature. Par ailleurs, dans la leçon que Mallarmé donne aux hommes de lettres dans "Solitude " (Divagations), la vanité d'un artiste est présentée comme une impulsion à dépasser les règles et chercher du nouveau (voir 2003, p. 258).

L'après-midi d'un faune permet de saisir encore une conséquence du processus de "creuser le vers ", à savoir la représentation typographique du vide. En effet, la crise du vers conduit à la séparation au sein de celui-ci et à l'instauration d'un vide entre des éléments jusque-là réunis (voir Gallardo, 1998, p. 14). L'opération d'espacer le vers qui, dans la structure du poème, fait apparaitre des moments de silence, à la manière des pauses en musique, a été pleinement réalisée dans Un Coup de dés. Par ailleurs, l'avant-dernière page du texte renvoie à "l'acte vide » qui met en cause l'authenticité de la réflexion poétique ce qui, encore une fois, fait preuve de la négativité, ce principe directeur de la création mallarméenne.

3. Il est intéressant que dans "Feuillet d'album », Mallarmé, tel un faune, évoque "ce vain souffle » de sa flûte poétique incapable de reproduire la réalité : le rire naturel d'un enfant. 


\section{5. Écrire des Vanités}

En analysant la vanité ou l'inanité mallarméenne, il faut indiquer également celle qui renvoie à l'impuissance créatrice du poète lui-même. Dans plusieurs lettres, il qualifiait son état d'esprit comme " parfaitement vide ", une conséquence des travaux scolaires qu'il était obligé de poursuivre, ou d'autres soucis quotidiens. Ce phénomène douloureux qui accompagnait Mallarmé au cours de toute sa vie, est abordé dans le poème "L'Azur " où l'âme vide du poète risque de succomber aux menaces de l'azur qui le guette comme un animal féroce. La stérilité de l'artiste, cette « impossibilité d'écrire » ou le vide spirituel, encore une fois s'avère fécond, car cette impuissance "paradoxalement devient sujet d'écriture " (Benoit, 2007, p. 39). Ainsi, dans la hantise de la page blanche, «le vide papier que la blancheur défend » («Brise marine », 1998, p. 15), peut-on voir les potentialités créatrices dont témoignent le mieux les précitées recherches du Coup de dés.

Par contre, il est intéressant de regarder sous cet angle les « Notes pour un tombeau d'Anatole " que Mallarmé avait rédigées après la mort de son fils, en vue d’en composer, par la suite, une sorte d'hommage à l'enfant emporté par une maladie mystérieuse à l'âge de huit ans. Parmi les réflexions éparses sur une feuille de papier, ces bribes de pensées douloureuses, se remarque le recours constant aux motifs du vide et de la vanité, ce qui permet de voir dans l’écriture mallarméenne plus qu'un aveu du père en deuil. À force de recréer l'expérience de la disparition d'Anatole, Mallarmé a mis en œuvre le concept et l'écriture des Vanités, ce topos bien connu dans la littérature et la peinture des XVI et XVII ${ }^{e}$ siècles, qui développe "la méditation sur la mort prochaine, la réflexion sur la fragilité de l'homme et des choses » (Verlet, 2001, p. 9). Conscient de passer les derniers moments avec son fils malade, le père évoque des "remèdes vains " face à la nature humaine, et des espaces qui bientôt se transformeront en symboles de la perte, comme la chambre d'enfant ou l'endroit où Anatole avait l'habitude de jouer. D'un côté, la conscience de la mort se trouve renforcée par la présence des objets appartenant au défunt, comme ses vêtements ou ses meubles. Mais de l'autre, Mallarmé élargit la sensation du vide créé par la disparition d'Anatole : un vide quasi physique, ressenti au sein de la famille en deuil qui vit le «temps de la chambre vide » (1998, p. 894), se double d'un «vide immense / produit par ce / qui serait sa vie» (1998, p. 893). Si la Vanité se fonde sur « une ontologie du rien », elle instaure, par conséquent, un nouveau rapport au temps où «l'être est réduit à son apparence momentanée ». Il s'agit d'un état transitoire, qui se fige " dans une sorte de présent éternel » (Kovàcs, 2008, p. 29). Telle semble être, en effet, la perception mallarméenne d'Anatole : malgré son absence, à travers les écrits du père où toutes les couches temporelles sont confondues, il se trouve placé toujours entre la vie et la mort, immobilisé dans le présent et projeté inutilement dans le futur au moyen des hypothèses irréelles.

Et pourtant, il est nécessaire de noter que par son entreprise, plutôt qu'à se rappeler la précarité de la vie, le père-poète visait à participer à la mort de son fils, 
recréant ainsi l'expérience du néant. Si Mallarmé renouvelle une mise à mort de son enfant, c'est pour y adhérer complètement, car la " fosse creusée par lui [Anatole] » (1998, p. 935) instaure de nouveau un rien que le poète doit transcender. L'écriture du vide laissé par la mort de l'enfant, de même que les tentatives de faire vivre celui-ci au moyen d'un ouvrage littéraire, conduisent à un autre vide, celui de la tombe (une image largement exploitée dans la représentation des Vanités ; voir Verlet, 2001, p. 8) qui illustre la mort du poète s'abolissant dans l'éparpillement de son texte 4 . À en croire Laurie Laufer, «le poète [...] qui se vit déjà mort, tente dans cette expérience poétique l'écriture de la disparition, l'écriture qui s'efface elle-même à mesure qu'elle s'inscrit» $(2009$, p. 98$)$.

" Je me sens couché en la tombe » (1998, p. 906), avoue Mallarmé, s’immergeant ainsi dans la mort, l'idée centrale de l'écriture des Vanités. En effet, l'enfant disparu qui semble mourir encore une fois et faire redécouvrir au père « le néant moderne » amené par un "vent de rien qui souffle " (1998, p. 901), devient un sujet et un objet du texte, même si le projet mallarméen de lui construire « le tombeau idéal » (1998, p. 939) n'est qu'illusoire. Que la tâche de dépasser la mort par le processus de spiritualisation du défunt dès le début soit vaine, étant donné la non-croyance mallarméenne en l'au-delà, il ne fait pas de doute que la recherche d'un sens après la mort de l'enfant, ou le repassage des vanités de la vie, s'identifient à une quête de la notion pure, cet objectif premier de toute écriture.

D’après Karine Lanini, l'originalité de la vanité réside dans le fait qu'elle constitue un objet du discours et un principe qui remet en cause les fondements du discours (2006, p. 13). Chez Mallarmé, parmi une multitude d'acceptions que revêt ce terme, il est pourtant possible de voir un renversement paradoxal de ce principe. Ayant constaté la vanité du langage, tout en admettant le caractère presque vaniteux de la poésie, il invente un moyen de dépasser ce constat pour, au prix de la décomposition du moi, transformer l'expérience existentielle du vide en bonheur de création, bien que celle-ci relève d'un mensonge. Ainsi, dans les notes réunies sous le titre "Épouser la notion ", résume-t-il l'évolution de sa pensée : "quand bien / même il n'en existerait rien - / j'y crois - / j'y crois / et il faut qu'il n'en / existe rien pour que / je l'étreigne et y / croit totalement " (1998, p. 1067-1068). Paul Valéry a noté dans ses Cahiers que, contrairement à l'orgueil, « la vanité est jouissance » (II, 1974, p. 1403). Mallarmé a finalement réussi, « derrière l'enseigne ironique de la littérature ", à jouir de sa pensée et de sa sensibilité, en y ajoutant le " plaisir d'amitié, de vanité, d'amour, plaisir - enfin, après de si tristes années - de la quotidienneté assumée »(Vaillant, 2005, p. 382).

4. Un sentiment de mort, toujours présent chez Mallarmé, relève aussi de ses problèmes de santé (insomnies, états nerveux) auxquels contribuait son travail intellectuel fatiguant, y compris la création poétique. C'est pourquoi, venant de découvrir le Néant, Mallarmé ne tarde pas à noter : "l'autre vide que j'ai trouvé, est celui de ma poitrine » ("À Henri Cazalis », 1998, p. 696). 


\section{RÉFÉRENCES}

Albrecht, F. (2016). L'objet musical chez Mallarmé, instrument des fuites. Dans A. Bonnet et P.-H. Frangne (dir.), Mallarmé et la musique, la musique et Mallarmé (p. 79-96). Rennes, France : PUR.

Anderson, J. (2002). La Poésie éprise d’elle-même. Poétique de Stéphane Mallarmé. New York, États-Unis : Peter Lang.

Bayle, C. (2014). Nocturne de l'âme moderne. Le Spleen de Paris de Charles Baudelaire. Rouen, France : PURH.

Bénichou, P. (1998). Entretien : Sens et obscurité selon Mallarmé. Magazine Littéraire, 368, 56-60.

Benoit, É. (2001). De la crise du sens à la quête du sens : Mallarmé, Bernanos, Jabès. Paris, France : Cerf.

Benoit, É. (2007). Néant sonore. Mallarmé ou la traversée des paradoxes. Genève, Suisse : Droz.

Bougault, L. (1998). L'esthétique de l'éventail : être, paraître et disparaître dans la poétique mallarméenne. Dans F.-Ch. Gaudard (dir.), Poésies. Stéphane Mallarmé (p. 61-79). Paris, France : Ellipses.

Davies, G. (1988). Mallarmé et la " couche suffisante d'intelligibilité». Paris, France : José Corti.

Delègue, Y. (2001). Mallarmé, le sujet de la poésie. Revue d'histoire littéraire de la France, 5(101), 1423-1432.

Ettlin, A. (2017). Le double discours de Mallarmé. Une initiation à la fiction. Paris, France : Ithaque.

Gallardo, J.-L. (1998). Mallarmé et le jeu suprême. Orléans, France : Paradigme.

Kovàcs, K. (2008). La Vanité au XVII ${ }^{\mathrm{e}}$ siècle : pouvoir de séduction et passage du temps. Dans Le Passé dans le Présent, le Présent dans le Passé (p. 25-30). Szeged, Hongrie : JATEPress.

Lanini, K. (2006). Dire la vanité à l'âge classique. Paradoxes d'un discours. Paris, France : Honoré Champion.

Laufer, L. (2009). La sépulture mallarméenne. Pour un tombeau d’Anatole. Cliniques Méditerranéennes, 2(80), 97-110.

Mallarmé, S. (1998). Euvres complètes. Vol. I. B. Marchal (éd.). Paris, France : Gallimard.

Mallarmé, S. (2003). Euvres complètes. Vol. II. B. Marchal (éd.). Paris, France : Gallimard.

Marchal, B. (1998). Mallarmé. Mémoire de la critique. Paris, France : PUPS.

Millan, G. (2014). Cent ans de critique mallarméenne : bilan et perspectives. Dans B. Marchal et J.-L. Steinmetz (dir.), Mallarmé ou l'obscurité lumineuse (p. 369-380). Paris, France : Hermann.

Proust, M. (1971). Contre Sainte-Beuve. P. Clarac et Y. Sandre (éd.). Paris, France : Gallimard. Vaillant, A. (2005). La crise de la littérature. Romantisme et modernité. Grenoble, France: ELLUG. Valéry, P. (1974). Cahiers. Vol. II. J. Robinson (éd.). Paris, France : Gallimard.

Verlet, A. (2001). Les Vanités de Chateaubriand. Genève, Suisse : Droz.

RÉSUMÉ : L'article analyse la notion polysémique de vanité dans la pensée et dans l'œuvre de Mallarmé. Le concept dévoile les paradoxes de la création mallarméenne : le poète jugé obscur et accusé de vanité, était déchiré entre le rêve de gloire et l'autodévalorisation. C'est dans l'Art auto-réflexif par excellence qu'il projetait sa satisfaction 
d'artiste et trouvait un sens de la vie. Mais ayant vécu une crise spirituelle et découvert la vanité du langage, Mallarmé a choisi de prolonger une illusion de la littérature en trouvant dans le Néant les ressources de sa poésie. À force de "creuser le vers", il construit donc une poétique de la vanité qui reste synonyme d'inanité ou de vide où le sens des mots est relayé par la sonorité du langage. En plus, tout en luttant contre la stérilité poétique, à travers l'écriture des Vanités, Mallarmé s'immerge dans la mort afin de créer un « tombeau idéal » pour son fils disparu.

Mots-clés : Mallarmé, vanité, néant, vide, mort

\section{Mallarmé and the paradoxes of vanity}

ABSTRACT: This article analyses a polysemous term of vanity in Mallarmé's idea and œuvre. This concept shows paradoxes of Mallarmé's creation: a misunderstood poet accused of being vain who was torn between the dream of glory and the self-devaluation. It was in the autoreflexive art par excellence, where Mallarmé saw his satisfaction and found the sense of life. After the experience of spiritual crisis and the discovery of worthlessness of language, Mallarmé, finding in the Nothingness the resources of poetry, decided to extend the illusion of literature. "Digging into the line", he built the poetics of vanity which is a synonym of futility or void where the sense of word is replaced by the language sonority. What is more, while fighting against the poetic impotence, Mallarmé, due to writing about Vanitas, immersed into death to create an "ideal tomb" for his late son.

Keywords: Mallarmé, vanity, nothingness, void, death 